\title{
Pallidal Neuron Activity Increases during Sensory Relay through Thalamus in a Songbird Circuit Essential for Learning
}

\author{
Abigail L. Person ${ }^{1}$ and David J. Perkel ${ }^{2}$ \\ ${ }^{1}$ Graduate Program in Neurobiology and Behavior and ${ }^{2}$ Departments of Biology and Otolaryngology, University of Washington, Seattle, Washington 98195
}

Disinhibition of the thalamus remains the primary model of information transfer between the basal ganglia and the cortex. Yet in apparent conflict with this model, the globus pallidus, a GABAergic basal ganglia output structure, often exhibits marked increases in firing rate during movement. To investigate the translation of pallidal activity and its relay through the thalamus, we explored a basal ganglia-thalamic pathway essential for song learning in songbirds. We found that single units in the thalamic nucleus DLM of urethaneanesthetized adult male zebra finches responded selectively to playback of the bird's own song, like neurons in its upstream and downstream nuclei. Because the pallidal input to these neurons forms giant calyx-like synapses, we were able to record extracellular signals from these presynaptic terminals as well. Pallidal units were distinctly excited by song playback, suggesting an increase in GABAergic transmission in the thalamus during sensory processing. However, this overall increased firing rate was phasic, punctuated by rapid decelerations in firing rate. In several cases, we were able to record presynaptic and postsynaptic units simultaneously. Correlating the presynaptic and postsynaptic activity, we found that disinhibition of thalamus may entail pallidal firing rate decelerations rather than simple long pauses in spontaneous activity, as has long been assumed.

Key words: thalamus; songbird; basal ganglia; pallidum; DLM; Area X

\section{Introduction}

The canonical hypothesis of basal ganglia information transfer through the thalamus states that pauses in spontaneous highfrequency (HF) inhibitory inputs "disinhibit" thalamic target neurons to permit spiking (Deniau and Chevalier, 1985). Although disinhibition is supported experimentally between the substantia nigra pars reticulata and the superior colliculus or the thalamus (Hikosaka and Wurtz, 1983; Deniau and Chevalier, 1985), neurons in the internal segment of the globus pallidus (GPi), the primary output structure of telencephalic basal ganglia in mammals, display a paradoxical increase in firing rate in relation to movement (Anderson and Horak, 1985; DeLong et al., 1985; Turner and Anderson, 1997). This finding is puzzling because it suggests that the thalamic targets of the GPi experience increased GABAergic inhibition during movement, precisely when thalamic targets are activated (Anderson and Turner, 1991).

GABA can have diverse effects on thalamic neurons, ranging from inhibition to excitation via postinhibitory rebound (von

\footnotetext{
Received Jan. 9, 2007; revised June 23, 2007; accepted June 26, 2007.

This work was supported by National Institutes of Health (NIH) Grants MH066128 (D.J.P.), F31 NS049732 (A.L.P.), and T-32 GM07108 (A.L.P.) and NIH-National Institute on Deafness and Other Communication Disorders P30 Core Grant DC04661. We are affiliates of the Virginia Merrill Bloedel Hearing Research Center. We thank Michael Burger for technical advice at initial stages of this project, Edwin Rubel for the use of laboratory equipment, Dr. Cliff Hume for use of laboratory space, and Brandon Warren for technical support. We are grateful to Fred Rieke, Michele Solis, Gabe Murphy, Michael Farries, and Sam Gale for helpful comments on this manuscript.

Correspondence should be addressed to Dr. David J. Perkel, Departments of Biology and Otolaryngology, University of Washington, 1959 Northeast Pacific Street, HSB BB1165, Box 356515, Seattle, WA 98195. E-mail: perkel@u.washington.edu.

DOI:10.1523/JNEUROSCI.2045-07.2007

Copyright $\odot 2007$ Society for Neuroscience $\quad 0270-6474 / 07 / 278687-12 \$ 15.00 / 0$
}

Krosigk et al., 1993; Steriade and Contreras, 1995). This complexity illustrates why understanding pallido-thalamic interaction is crucial for interpreting how basal ganglia information is transferred to the cortex. However, little is known about mammalian pallido-thalamic cellular processing. Conversely, synaptic properties of a similar pallido-thalamic connection in an emerging model of basal ganglia thalamocortical function, the songbird anterior forebrain pathway (AFP), have been studied (Luo and Perkel, 1999b; Person and Perkel, 2005) and may therefore help illuminate general principles of basal ganglia relay (Doupe et al., 2005) (see Fig. 1).

The AFP is required for song learning in juvenile birds and for vocal plasticity in adults (see Fig. 1A) (Bottjer et al., 1984; Sohrabji et al., 1990; Scharff and Nottebohm, 1991; Williams and Mehta, 1999; Brainard and Doupe, 2000a,b; Kao et al., 2005). The basal ganglia component of this circuit, Area X, displays motorrelated activity in behaving animals and selective auditory responses in anesthetized animals (Doupe and Konishi, 1991; Hessler and Doupe, 1999). Area X contains GABAergic, pallidal-like projection neurons, which powerfully inhibit thalamic target neurons in the medial portion of the dorsolateral nucleus of the anterior thalamus (DLM) (Luo and Perkel, 1999a,b; Farries et al., 2005; Person and Perkel, 2005).

The striking electrophysiology of this synapse is supported by an anatomical specialization in which each thalamic neuron receives only one, or at most two, giant perisomatic terminals from a pallidal input (Fig. 1 B) (Luo and Perkel, 1999a,b). This arrangement of the presynaptic and postsynaptic cells in DLM allows direct exploration of pallido-thalamic input-output functions. 


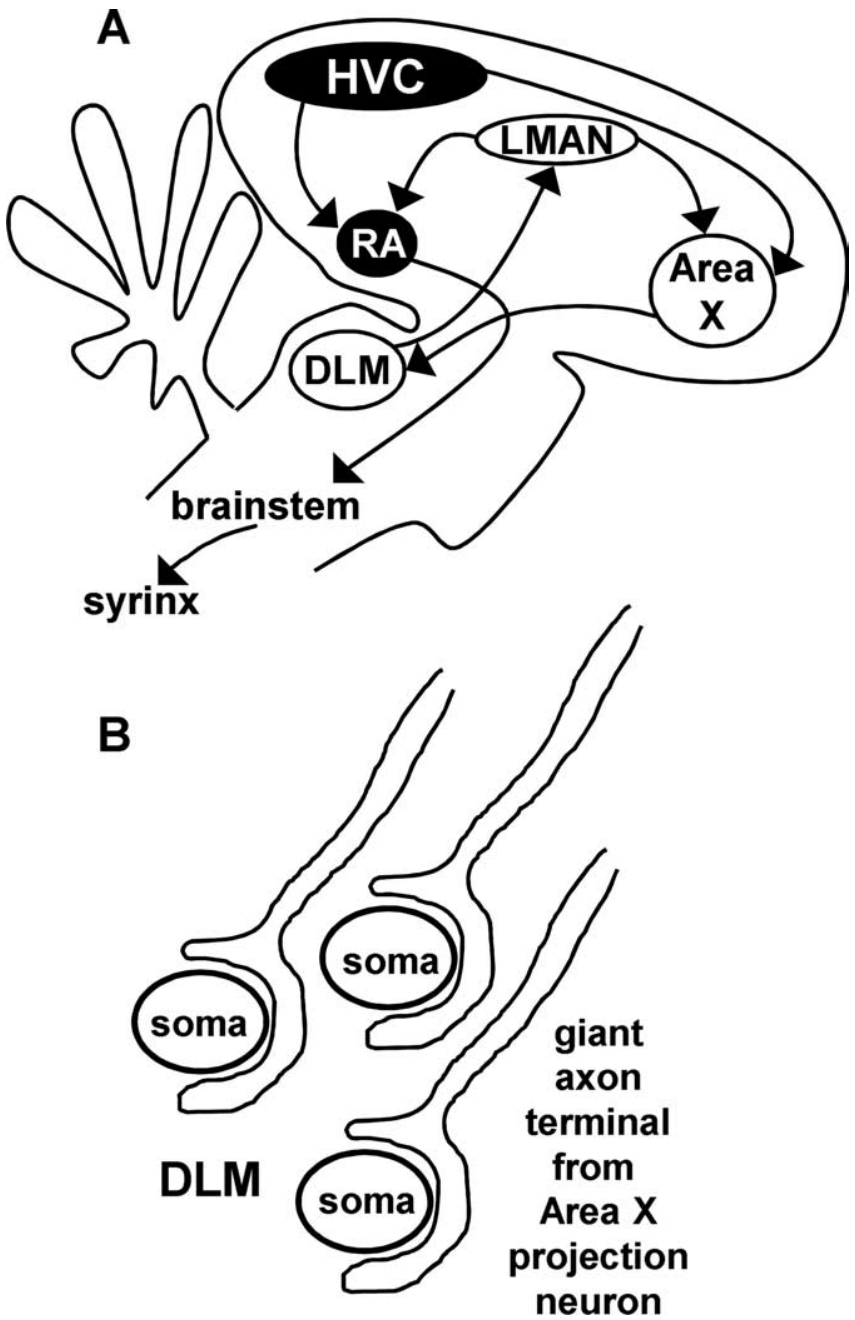

Figure 1. Neural circuitry of the song-control system and specialized pallido-thalamic synapse in DLM. $A$, Schematic diagram of oscine song-control nuclei. Nuclei in black comprise an obligatory motor pathway for song. Nuclei in white comprise the AFP required for juvenile song learning and adult vocal plasticity. $\boldsymbol{B}$, Schematic representation of the giant one-to-one GABAergic synapse from the basal ganglia structure Area X onto DLM somata.

In vitro, DLM neurons fire during irregular HF pallidal IPSP barrages, responding to short-duration pauses $(\sim 30 \mathrm{~ms}$ in duration) or rapid decelerations in IPSP rates with postinhibitory rebound spikes (Person and Perkel, 2005).

To examine further how the thalamus processes pallidal inputs, we recorded from thalamic targets of pallidal neurons in vivo. DLM somata displayed selective auditory responses to playback of the bird's own song (BOS), as observed in nuclei upstream and downstream of DLM (Doupe and Konishi, 1991). Unexpectedly, we also recorded from giant pallidal nerve terminals in DLM originating from Area X somata, which were also activated by song. Paired presynaptic and postsynaptic recordings revealed the pattern of pallidal input preceding thalamic firing in vivo. Pallidal activity often increased briefly just before a rapid deceleration preceding thalamic spiking. These data show that disinhibition may involve patterns of pallidal firing not previously appreciated.

\section{Materials and Methods}

Data from 44 adult male zebra finches (Taeniopygia guttata) were used in this study in accordance with an animal use protocol approved by the Institutional Animal Care and Use Committee at the University of Wash-
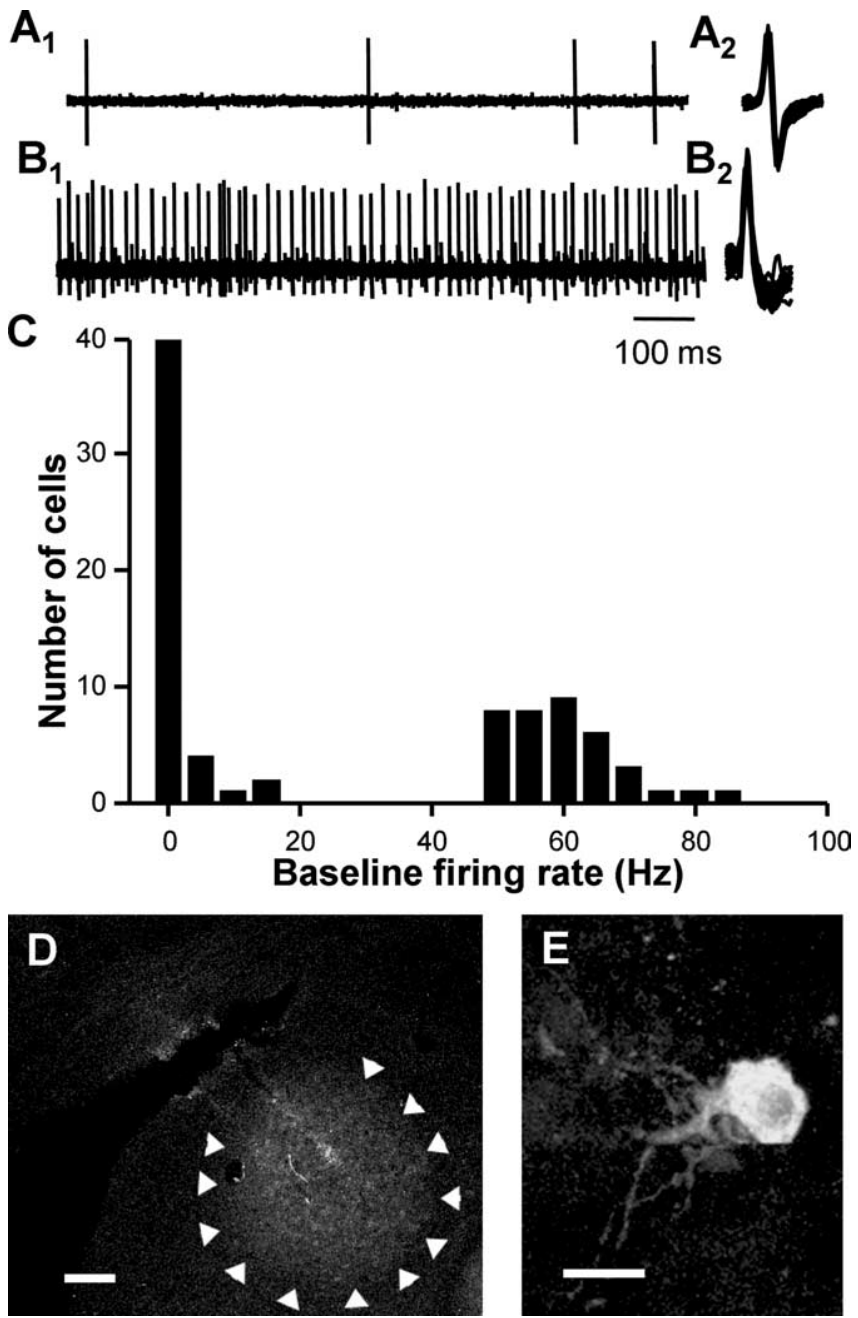

Figure 2. DLM neuron background activity patterns and recording site confirmation. $A_{1}, \mathrm{ALF}$ unit with biphasic action potential waveform in DLM. The time scale is as in $\boldsymbol{B}_{1} . \boldsymbol{A}_{\mathbf{2}}$, Overlaid spike waveforms. $\boldsymbol{B}_{1}$, A HF unit with monophasic action potential waveform encountered in DLM. $\boldsymbol{B}_{2}$, Overlaid spike waveforms. $\boldsymbol{C}$, Histogram of baseline firing rates for all units recorded in DLM distinguishes at least two populations of cells. Bin size, $5 \mathrm{~Hz}$. D, Example of restricted dye injection within DLM after electrophysiological recording. Arrowheads indicate the border of DLM. Scale bar, $100 \mu \mathrm{m}$. $\boldsymbol{E}$, High-power image showing a soma labeled after juxtacellular labeling of an LF unit. Scale bar, $20 \mu \mathrm{m}$.

ington. Animals were obtained from a commercial supplier and housed under a 13/11 h light/dark cycle with food and water available ad libitum.

\section{Song recording and stimuli}

Before the experiment day, the subject was placed in a sound-attenuation chamber in the presence of a female zebra finch. His song was digitally recorded at $44 \mathrm{kHz}$ and edited to $1.7-3.2 \mathrm{~s}$ long, which included two or three stereotyped motifs for use as the BOS playback stimulus. This song was reversed in time to generate a reversed-BOS (Rev) stimulus with Goldwave (St. Johns, Newfoundland, Canada) sound editing software.

During electrophysiological recording, song stimuli were presented from a loudspeaker positioned $0.3 \mathrm{~m}$ in front of the bird at amplitudes ranging from 65 to $78 \mathrm{~dB}$ peak sound pressure level. BOS and Rev song stimuli were interleaved randomly and presented every $15 \mathrm{~s}$.

\section{Surgery}

Each animal was food deprived for $1-2 \mathrm{~h}$ before beginning anesthesia. The animal was weighed and given an intramuscular injection with a total of $5.5-6.6 \mathrm{ml} / \mathrm{kg}$ of $20 \%$ urethane divided into three to four injections over the course of $90 \mathrm{~min}$. The animal was then placed in a felt restraining coat. A bolus of local anesthetic ( $1 \%$ lidocaine) was injected 

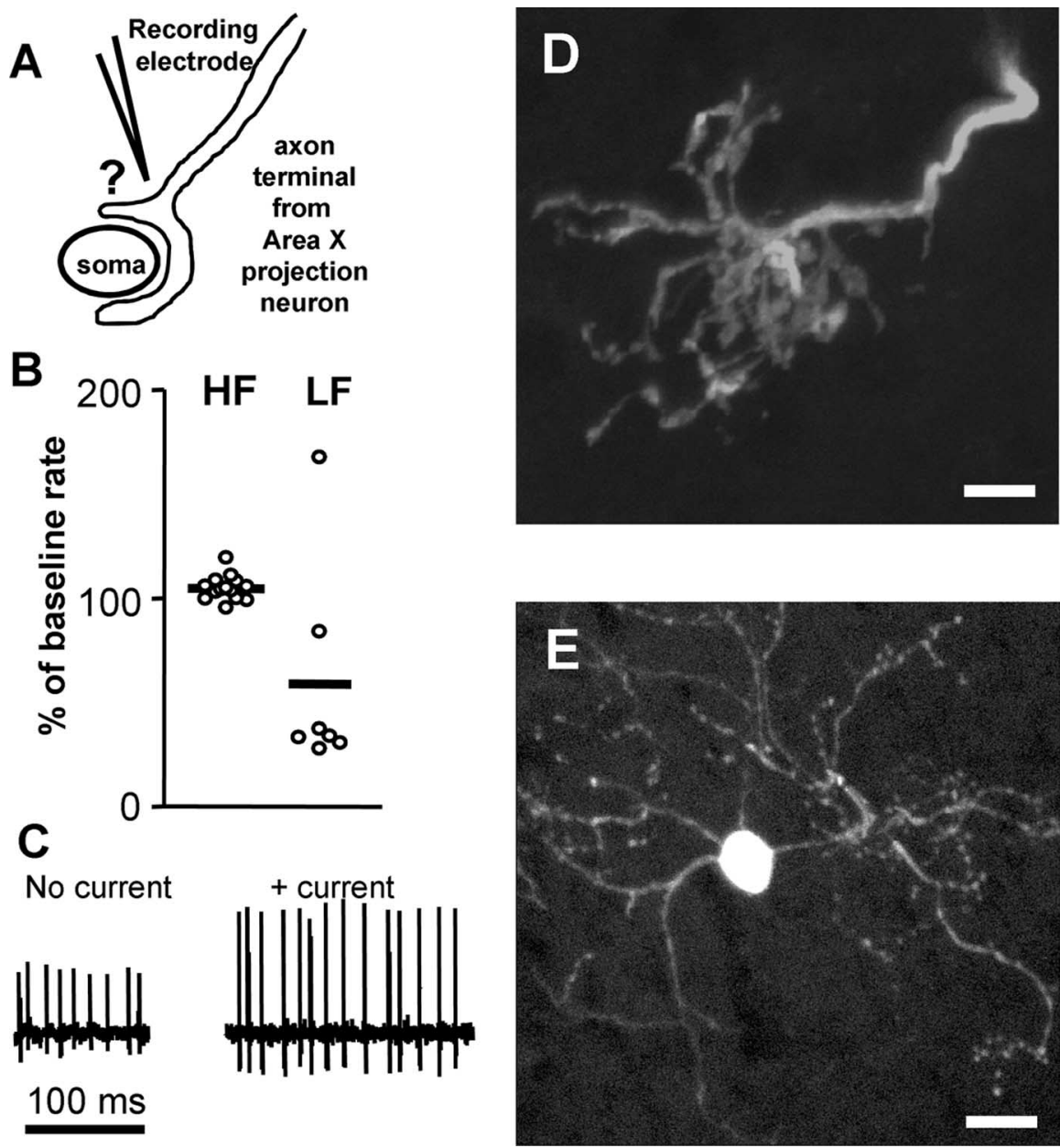

Figure 3. HF units in DLM are axon terminals from Area X. $\boldsymbol{A}$, Schematic representation of a hypothesized recording target, where the electrode is close to a giant synaptic terminal from a cell originating in Area X, forming a synapse in DLM. $\boldsymbol{B}, \mathrm{HF}$ units $(>40 \mathrm{~Hz})$ in DLM are insensitive to application of the $\mathrm{GABA}_{\mathrm{A}}$ receptor agonist muscimol. Baseline rates of LF units in DLM were most often reduced during muscimol application. $C$, Example of a change in signal-to-noise ratio as a result of applying a positive current ( $\sim 5 \mathrm{nA}$ ) while recording an $\mathrm{HF}$ unit. $\boldsymbol{D}$, A giant axon terminal typical of Area X-to-DLM projection neurons was revealed after juxtacellular labeling of an HF unit in DLM. $\boldsymbol{E}$, A soma labeled in Area X after HF-unit juxtacellular labeling in DLM. The DLM HF-unit labeling filled a single axon that could be followed back to Area $X$ where an individual large soma with aspiny dendrites was labeled. Scale bars: $\boldsymbol{D}, \boldsymbol{E}, 20 \mu \mathrm{m}$.

subcutaneously under the scalp just before the animal was placed in the head holder of a stereotaxic apparatus. A heating blanket was used continuously during the experiments. A small craniotomy was made above the midline reference point, the bifurcation of the midsagittal sinus, and small craniotomies were made above DLM bilaterally. A post was then glued to the skull to secure the head without ear bars. The long-lasting local anesthetic bupivacaine was applied to the incision at $3 \mathrm{~h}$ intervals during the course of the experiment. The stereotaxic apparatus and recording equipment were housed inside a double-walled walk-in sound attenuation booth (Industrial Acoustics, Bronx, NY).

\section{Electrophysiological recording}

Electrodes. Glass pipettes (A-M Systems, Carlsborg, WA) were pulled on a vertical micropipette puller (model 700C; David Kopf Instruments, Tujunga, CA), and the tips were blunted to achieve electrode impedances between 1 and $5 \mathrm{M} \Omega$. For experiments targeting paired presynaptic and postsynaptic elements, electrode impedances were higher: between 6 and $50 \mathrm{M} \Omega$ (see Results). Electrode tips were filled with 5\% Alexa-488- or -568-conjugated $10 \mathrm{kDa}$ dextran amine (Invitrogen, Eugene, OR) in $1 \mathrm{M}$ phosphate buffer, and the electrode shaft was then backfilled with $2 \mathrm{M}$ $\mathrm{NaCl}$. In all recordings, a ground electrode was placed in the cerebellum, posterior to the decussation of the midsagittal sinus.

For iontophoretic drug application, "piggy-back" multibarrel mi- cropipettes were fashioned as described by Havey and Caspary (1980). A triple-barrel blank was pulled on a vertical puller and blunted. The single electrode tip was then secured 2-20 $\mu \mathrm{m}$ beyond the tip of the multibarrel tip with cyanoacrylate glue and dental cement. Two of the three "drug" barrels were filled with $8 \mathrm{~mm}$ muscimol in $0.9 \% \mathrm{NaCl}, \mathrm{pH} 5$. The final barrel was filled with $2 \mathrm{M} \mathrm{NaCl}$ and served as a balance to ground. An ION-100T iontophoresis current source (Dagan, Minneapolis, MN) was used for drug injection.

Recordings. Single-unit extracellular recordings from DLM were obtained using BOS alone or BOS and Rev interleaved as search stimuli. Only units with a signal-to-noise ratio over 3:1 and uniform waveforms were included in the dataset. Fifty-nine of 84 units had signal-tonoise ratios over 6:1. Neuronal spike times were stored using custom-written data acquisition software (B. Warren, University of Washington, Seattle, WA). Signals were amplified 10fold with a Neuroprobe amplifier (model 1600; A-M Systems), low-pass filtered at $5 \mathrm{kHz}$, passed through a Hum Bug noise eliminator (AutoMate Scientific, San Francisco, CA), amplified further 10- to 100-fold, and filtered (300 $\mathrm{Hz}$ high-pass, $3 \mathrm{kHz}$ low-pass) with a Tucker Davis Technologies (Alachua, FL) spike conditioner. Recordings were monitored on an oscilloscope. Event time stamps were collected with $1 \mu$ s resolution using a Tucker Davis Technologies window discriminator. For most recordings, waveform data were also sampled at 20 $\mathrm{kHz}$.

At 11 sites, pairs of auditory units with distinct waveforms were recorded simultaneously. For these recordings, spikes were isolated offline using Spike2 software (Cambridge Electronic Design, Cambridge, UK). Waveforms of paired presynaptic and postsynaptic spikes (see Results) differed in amplitude by a factor of at least 10:1, permitting unambiguous classification of spikes into two categories using template-matching spike sorting. For sorting, trigger thresholds were set at twice the level of the noise, and template windows were $<1 \mathrm{~ms}$ in width (mean, $0.54 \mathrm{~ms}$; range, $0.3-0.7 \mathrm{~ms}$ ). In several cases, we manually corrected overclustering of presynaptic spikes to reflect a single unit within a single cloud of principal components. Relatively low-amplitude presynaptic potentials have been detected and studied in the lateral geniculate nucleus (LGN) (Hubel and Wiesel, 1961; Kaplan and Shapley, 1984; Wang et al., 1985; Sincich et al., 2007) and ascending auditory system (Kopp-Scheinpflug et al., 2002), lending support to the notion that we recorded from synaptically connected pairs of neurons. We calculated autocorrelations for HF units in paired recordings, and in all cases, the extracted units obeyed a refractory period of $1 \mathrm{~ms}$.

\section{Anatomy}

We monitored electrode placement in three ways. Gross observation of electrode tracts was possible because DLM is located $\sim 4 \mathrm{~mm}$ below the dorsal surface of the brain. In addition, we loaded our recording pipettes with the fluorescent neuronal tracer Alexa 488-conjugated $10 \mathrm{kDa}$ dextran amine. We were able to recover dye deposits after recording in 27 animals. Dye could be bulk ejected by passing 3-20 nA positive current continuously through the recording pipette for $2-20 \mathrm{~min}$. In addition, we labeled neurons juxtacellularly. In these experiments, we recorded from a single unit isolated such that we could drive its activity or alter the spike signal-to-noise ratio with a small continuous current injection $(<5 \mathrm{nA})$ 
A
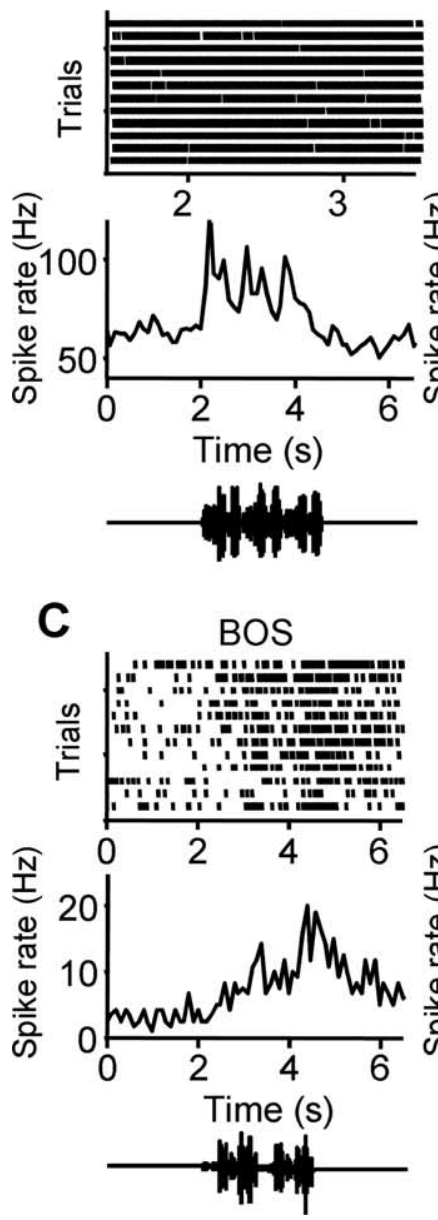

Reversed
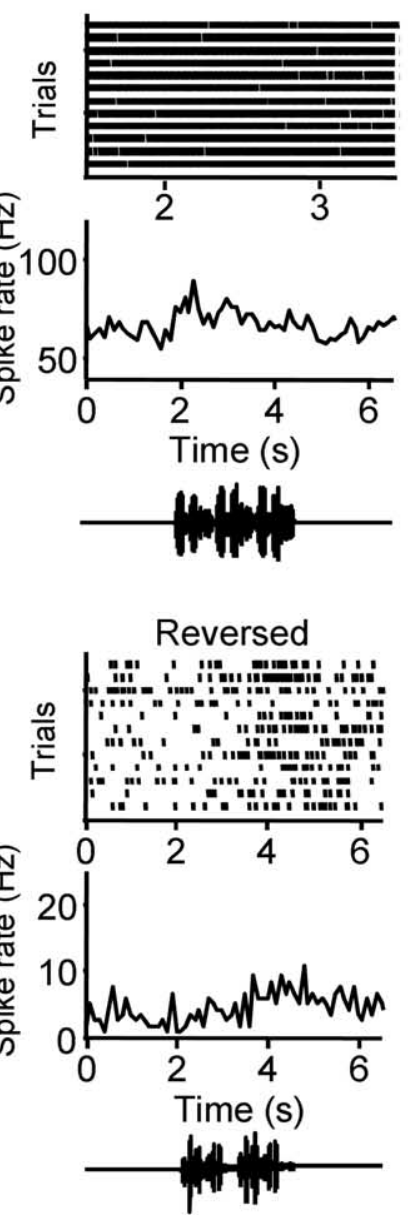

B

BOS
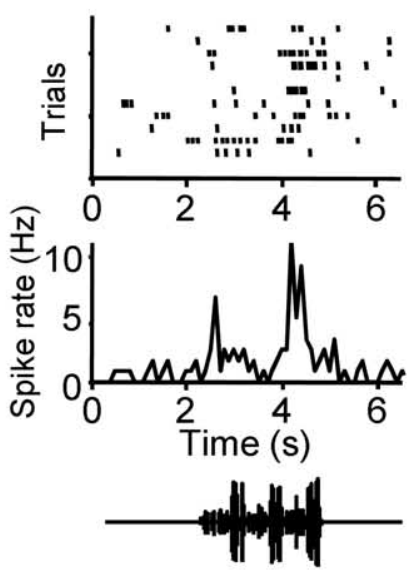

D

BOS

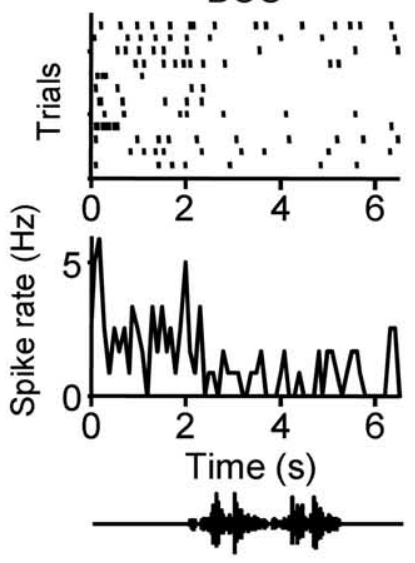

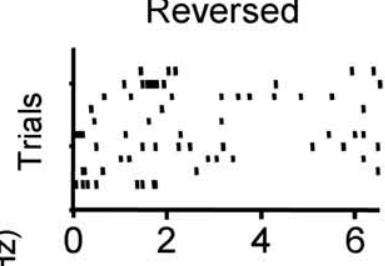

Reversed
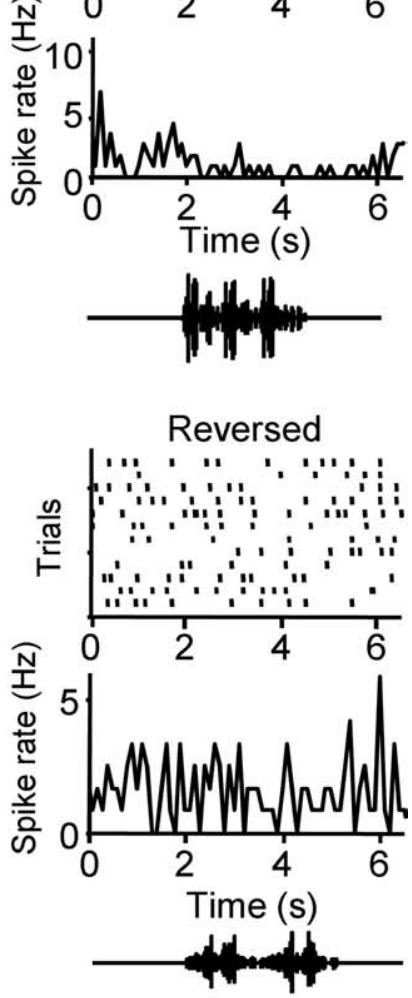

Figure 4. DLM neurons respond to playback of the BOS. $A$, Raster plot and peristimulus time histogram illustrating response of an HF unit to playback of BOS and Rev indicated in oscillograms in the bottom panel. $\boldsymbol{B}$, Raster plot and peristimulus time histogram illustrating the auditory responses of an LF unit in DLM. C, An example of a unit in DLM with auditory responses that persisted after song playback had ceased. $\boldsymbol{D}$, An example of a unit in DLM that was inhibited during song playback.

for $>1$ min. This process drives dye into the cell (Pinault, 1996), and post hoc observation revealed a single-cell label.

For visualizing electrode tracts, recording sites, or juxtacellular label, tissue was fixed in $4 \%$ paraformaldehyde for $24 \mathrm{~h}$. The brain was then cryoprotected in $30 \%$ sucrose, cut to $40-\mu \mathrm{m}$-thick sections in the parasagittal plane on a freezing microtome, and processed for fluorescence microscopy. Images were acquired using an Olympus (Tokyo, Japan) Fluoview laser-scanning confocal microscope and were processed for brightness and contrast in Photoshop 7.0 (Adobe Systems, San Jose, CA).

\section{Data analysis}

Event times were analyzed for responses during auditory stimuli using Matlab 7.0.1 software (MathWorks, Natick, MA). For each cell, we calculated baseline firing rate, firing rate during stimulus playback, the interspike interval (ISI) distribution, significance of auditory response, response strength (RS), and $d^{\prime}$, a metric of selectivity (see below for details) (Green and Swets, 1966; Solis and Doupe, 1997). Any unit with an average firing rate during BOS playback that was significantly different from the baseline rate ( $p<0.05$, two-tailed $t$ test) coupled with histological evidence that the recording site was within DLM was included in the dataset. Baseline firing rate was calculated during the $2 \mathrm{~s}$ epoch before song playback (Rosen and Mooney, 2000). Firing rate during playback was calculated from spikes that occurred during the stimulus. The difference between the firing rate (in hertz) during a given stimulus and during the $2 \mathrm{~s}$ baseline period was calculated for each trial and averaged across trials to give a mean RS. The RS reflects the average difference in firing rate between baseline and playback periods.
RS measurements were used to calculate the discriminability statistic $d^{\prime}$, which is used to quantify the selectivity of a neuron for a given stimulus over another (Green and Swets, 1966), where

$$
d^{\prime}{ }_{B O S-R e v}=\frac{(\sqrt{2})\left(\overline{R S}_{B O S}-\overline{R S}_{R e v}\right)}{\sqrt{\sigma_{B O S}^{2}+\sigma_{R e v}^{2}}}
$$

The difference between the average RSs to two stimuli was normalized by the square root of the average of the variances of the RS measurements for the two stimuli. High absolute values of $d^{\prime}$ indicate that the neuron consistently responded more to one stimulus than to another. Note that this formula differs from previous calculations of $d^{\prime}$ by a factor of $\sqrt{ } 2$ (Solis and Doupe, 1997, 1999; Janata and Margoliash, 1999; Rosen and Mooney, 2000); our calculation measures the ability of the RS of the neuron to determine whether a stimulus was BOS or Rev based on a single playback trial (Green and Swets, 1966).

\section{Pallidal deceleration analysis}

Decelerations in the firing rate of a class of units, those with high frequency (see Results), were located using the following analysis procedure. We took the instantaneous firing rate of each BOS-playback trial (the reciprocal of ISIs) and smoothed it with a Gaussian window with a width at half-maximal amplitude of $4 \mathrm{~ms}$ to diminish the contribution of single ISIs to the analysis. We next took the derivative of the smoothed rate function and calculated the value of its fifth percentile, using this as a threshold for detection of deceleration (see Fig. 6). Points where the derivative crossed the fifth percentile threshold in the downward direc- 
A

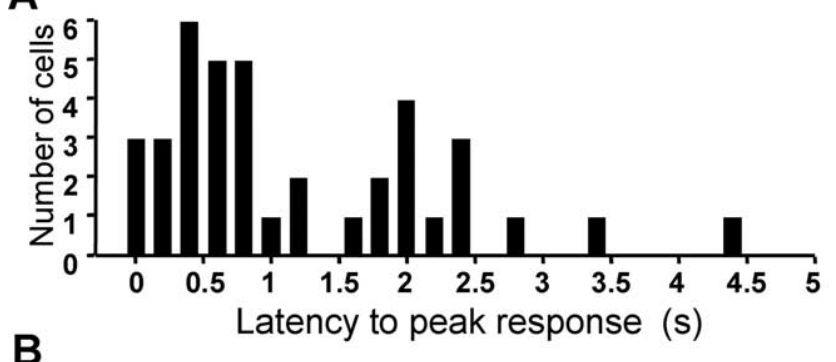

B

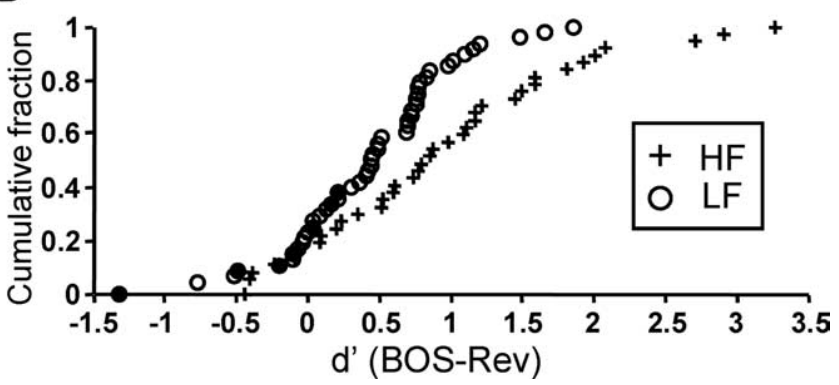

Figure 5. Properties of auditory responses in DLM. $\boldsymbol{A}$, Of the units with positive RS, the latency of the peak response to song varied but occurred primarily within 2.5 s from song onset. Bin size, 0.15 s. $B$, Cumulative distribution of $d^{\prime}$ measurements for discriminability between BOS and Rev playback for each unit. + , HF units; open circles, LF units; filled circles, LF units inhibited by song.

tion (indicating rapid deceleration) were indexed and used to calculate the time of decelerations for each sweep.

\section{Spike-triggered averaging}

For paired recordings, in which an axon terminal and soma were recorded simultaneously, we calculated a spike-triggered average (STA) to describe the pattern of axon terminal spiking before soma spikes. In these cases, axon terminal spike times in a $250 \mathrm{~ms}$ window before each soma spike were recovered and aligned relative to the time of the soma spike. The occurrence of axon terminal spikes was expressed as an average firing rate. To calculate the STA, the value for each bin in the histogram was divided by the number of soma spikes and multiplied by 1000/binwidth to recover the average rate of axon terminal spikes before the soma spike.

\section{Results}

\section{Spontaneous activity of DLM neurons in vivo}

We recorded from 84 single units in the thalamic nucleus DLM of urethane-anesthetized adult male zebra finches. Based on spontaneous firing rates, we encountered two classes of units in DLM. The first class had very low-frequency (LF) spontaneous firing rates (mean \pm SEM, $2.6 \pm 0.6 \mathrm{~Hz} ; n=47$ ) and biphasic actionpotential waveforms (Fig. $2 A_{1}, A_{2}$ ). The second unit class displayed spontaneous activity at much higher frequencies (mean, $62.2 \pm 1.37 \mathrm{~Hz} ; n=37$ ) with a characteristic monophasic action potential waveform (Fig. $2 B_{1}, B_{2}$ ). Notably, the presence of these HF units distinguished DLM from surrounding thalamic regions. Two non-overlapping spontaneous firing-rate distributions indicated at least two populations of cells: those with spontaneous firing rates above $40 \mathrm{~Hz}$, termed high frequency, or below $25 \mathrm{~Hz}$, termed low frequency (Fig. 2C). Post hoc histological examination confirmed that units displaying these spontaneous activity patterns were comingled within DLM (Fig. 2D).

We further characterized units within DLM using juxtacellular labeling to visualize the specific cell from which we recorded (Pinault, 1996). Juxtacellular labeling of an LF unit revealed a soma within the nucleus DLM (Fig. $2 E)(n=12)$. The neuronal morphology of labeled neurons resembled that of DLM neurons projecting to lateral magnocellular nucleus of the anterior nidopallium (LMAN) (Luo et al., 2001; Luo and Perkel, 2002).

\section{HF units are giant axon terminals originating from cells in Area $\mathrm{X}$}

Intrinsic properties of DLM neurons have been reported previously in slice studies (Luo and Perkel, 1999b, 2002). Under these conditions, HF spontaneously active neurons were never observed. We therefore wondered about the identity of the HF cells we recorded in DLM in vivo. Extracellular activity from giant axon terminals has been reliably detected in the ascending auditory system and the LGN (Hubel and Wiesel, 1961; Kaplan and Shapley, 1984; Wang et al., 1985; Kopp-Scheinpflug et al., 2002; Sincich et al., 2007). Thus, we hypothesized that the HF cells we recorded were axon terminals originating from output neurons of Area X (Fig. 3A).

To determine whether HF cells were axon terminals or somata, we reasoned as follows. Activation of $\mathrm{GABA}_{\mathrm{A}}$ receptors on a neuronal soma or dendrites should alter (usually reduce) the rate of action potential firing. On the other hand, activation of $\mathrm{GABA}_{\mathrm{A}}$ receptors on axon terminals of a spontaneously active cell should not alter the rate of action potential firing, even if $\mathrm{GABA}_{\mathrm{A}}$ receptors are present on those terminals, because the terminal is distal to the spike initiation zone.

We therefore tested the sensitivity of DLM units to application of the $\mathrm{GABA}_{\mathrm{A}}$ receptor agonist muscimol (Fig. $3 B$ ). We focally applied muscimol, $\mathrm{pH} 5$, iontophoretically while recording from units in DLM. Focal application of muscimol to LF units slowed ongoing firing rates in six of seven cases (Fig. 3B) (mean, $59.0 \pm$ $21.1 \%$ of baseline), demonstrating that the technique worked in our hands. In contrast, in 15 of $15 \mathrm{HF}$ cells, the spontaneous firing rate remained constant during application of muscimol (Fig. 3B) (mean, $104.7 \pm 1.5 \%$ of baseline). This finding is consistent with HF units being axon terminals.

As an additional test of the identity of HF units in DLM, we juxtacellularly labeled the cellular structure from which we recorded. Unlike passing current to LF cells, which could drive activity, passing current to HF units did not cause the unit to change its discharge rate but instead consistently increased the signal-to-noise ratio (Fig. 3C) (Pinault, 1996). On histological examination after iontophoretic dye injection into HF units, we observed dye-filled giant axon terminals typical of Area X-toDLM synapses (Fig. 3D) ( $n=27$ terminals in 12 hemispheres). In three cases, we were able to follow the filled single axon back into Area $\mathrm{X}$ in the telencephalon, where a large soma with aspiny dendrites typical of a pallidal-like aspiny fast-firing (AF) projection neuron was filled (Fig. 3E) (Farries and Perkel, 2002).

In summary, we found that rates of HF units in DLM remained unchanged in the presence of muscimol, and histological examination revealed giant synaptic terminals typical of those from Area X projection neurons forming synapses in DLM. Together, these data strongly suggest that HF units recorded in DLM are synaptic terminals of pallidal neurons, the somata of which are located in Area X.

\section{Neural responses of units in DLM to playback of song}

AFP neurons exhibit highly tuned responses to playback of BOS, responding more strongly to it than to other stimuli (Doupe and Konishi, 1991). Previous reports focusing primarily on other song-control nuclei have included brief accounts of DLM neuron responses in vivo (Okuhata and Saito, 1987; Doupe and Konishi, 1991). We extend those observations here.

Both HF and LF cell populations in DLM responded robustly 
A
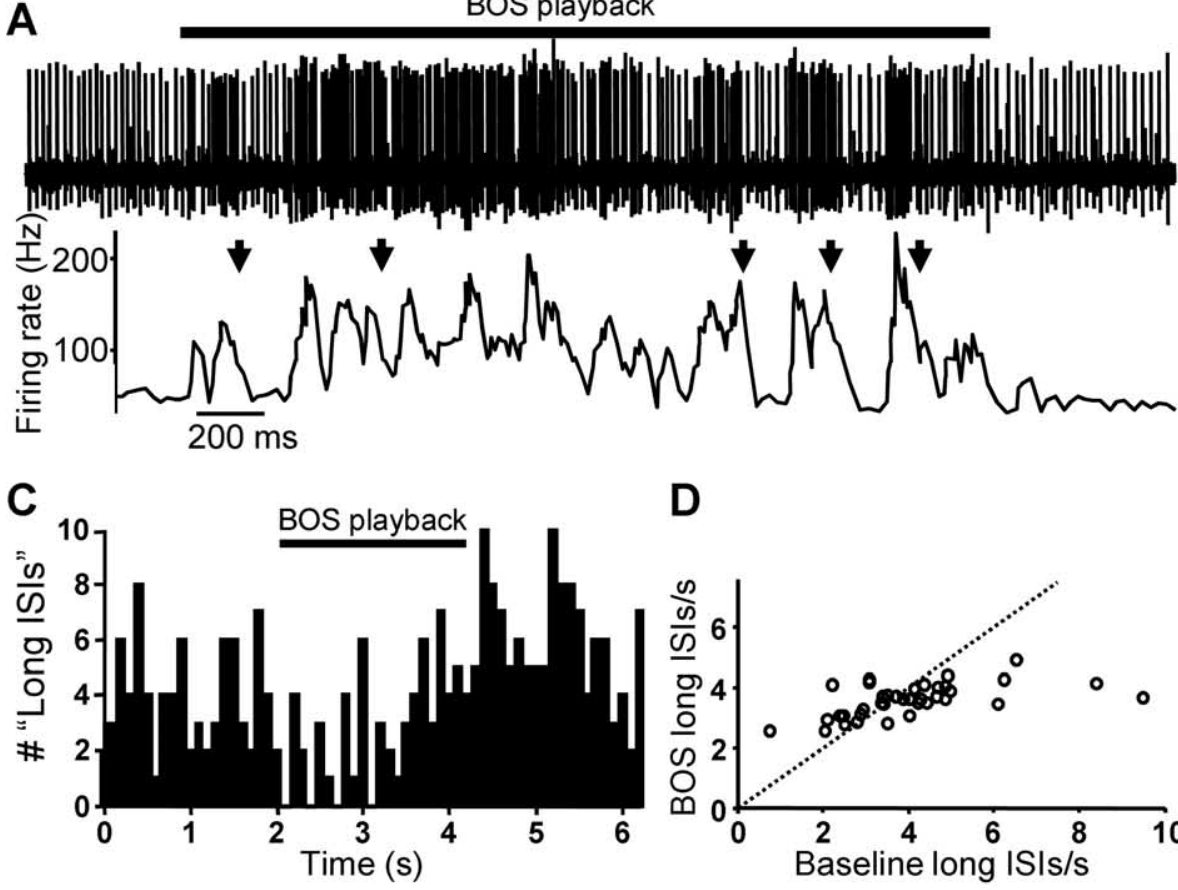

$\mathbf{F}$

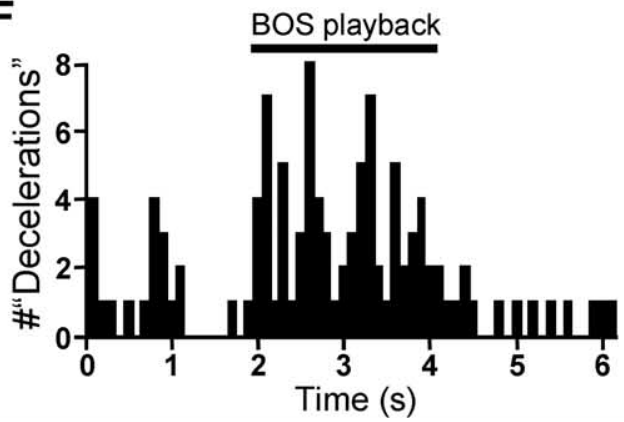

D

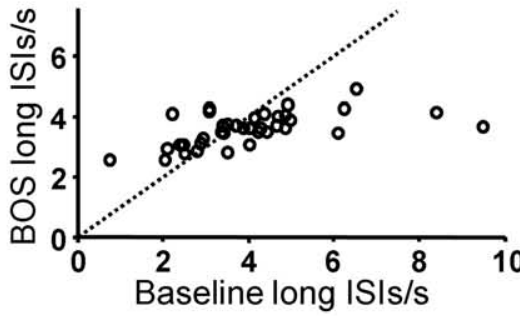

G

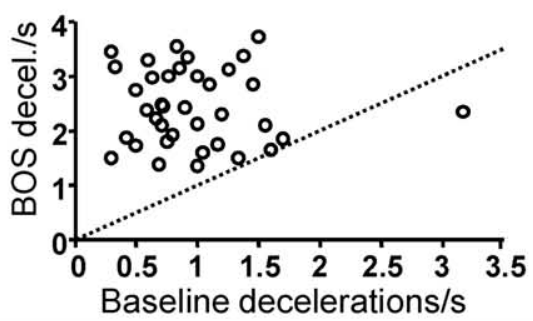

B
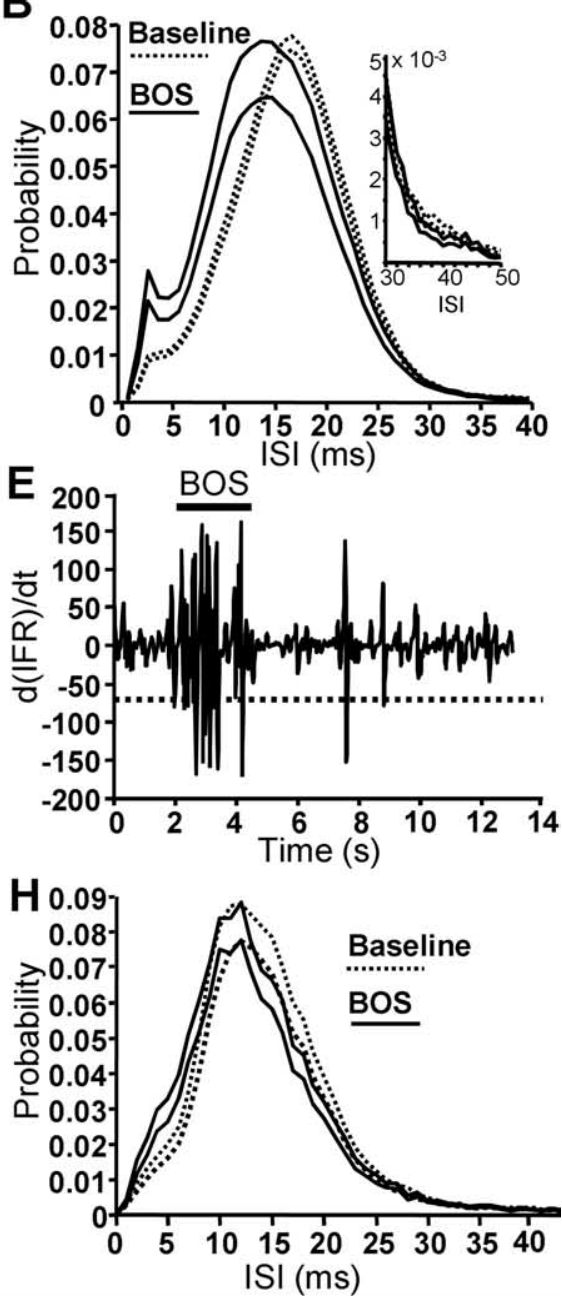

Figure 6. HF units exhibit rapid rate fluctuations during song playback. $\boldsymbol{A}$, Example of $\mathrm{HF}$ unit activity during a single trial of BOS playback. The spike rate function below the raw trace illustrates the phasic increases and decreases in rates typical of HF units. Arrows indicate epochs of rapid rate deceleration. $\boldsymbol{B}$, Probability distribution of ISIs during baseline (dotted line) and BOS (solid line) for all HF units. Each pair of lines represents mean and mean +1 SD. Inset, Right-hand tail of ISI probability distribution, demonstrating no difference in relative frequency of long intervals. $C$, An example of occurrences of long ISIs over multiple trials of BOS playback, defined as those ISIs above the 95th percentile of ISIs. D, Population summary of the rate of occurrence (occurrences per second) of long ISIs between BOS and baseline epochs. Each circle represents an HF cell. $\boldsymbol{E}$, An example of the derivative of a smoothed instantaneous firing rate (IFR) function ( $d$ (IFR)/dt) plotted against time. The dashed line indicates the fifth percentile used as a cutofffor defining rapid decelerations. $\boldsymbol{F}$, An example of the occurrence of decelerations in an example $H F$ cell across multiple trials of BOS playback (same cell as in C). The times of the $5 \%$ most extreme rate drops were measured with respect to song. Rate decelerations consistently occurred more frequently during BOS playback. G, Population summary of the rate (occurrences per second) of rapid decelerations during baseline periods is plotted against the number of rapid decelerations observed during BOS playback for each $\mathrm{HF}$ unit. Most units fall above the unity line, indicating more frequent decelerations during BOS playback than during baseline periods. $\boldsymbol{H}$, ISI probability distribution for $\mathrm{HF}$ units ( $n=11$ ) that were paired with LF neurons (Fig. 7) during baseline and BOS-playback epochs. Line conventions are the same as in $\boldsymbol{B}$.

to the playback of BOS (Fig. 4). Figure 4 illustrates typical responses to BOS and Rev with most units increasing discharge during the playback of BOS. Auditory responses tended to be selective for the song in the forward direction as seen by comparing peristimulus time histograms of responses to BOS and Rev (Fig. 4) (quantified below).

HF neurons exhibited phasic increases in firing rate during BOS playback and much smaller rate increases during Rev (Fig. $4 A$ ). Across the population of HF cells, RS to BOS playback was significantly greater than to playback of the reversed song (mean BOS RS, $9.3 \pm 1.2 \mathrm{~Hz}$; mean Rev RS, $3.4 \pm 0.8 \mathrm{~Hz} ; p<0.0001$, paired $t$ test). In fact, the rates and response profiles of HF cells in DLM closely resembled a class of HF neuron that has been recorded in Area X (Doupe, 1997), consistent with the finding that these units are axon terminals from Area X.

LF neurons qualitatively exhibited a range of auditory response profiles. Figure $4 B$ illustrates an example of the most commonly encountered LF cell response in DLM, with robust, selective responses to playback of BOS that occurred during song playback. Figure $4 C$ illustrates a second response profile of LF units in DLM. Here, the cell responded during the song, but the neural response continued after the song playback was complete. Occasionally $(n=7)$, song playback reduced spike rate in LF DLM neurons (Fig. 4D).

We quantified both RS and the latency of the peak response to song playback to illustrate the range of responses to song playback seen in auditory DLM neurons. RSs in LF units ranged from negative to positive, with a majority responding with increased rates during song playback (mean RS, $2.79 \pm 0.63 \mathrm{~Hz}$; range, -5.9 to17.9 Hz; mean peak rates, $12.57 \pm 2.07 \mathrm{~Hz}$ ). For LF cells with positive RSs, we measured the latency from song onset at which the average firing rate of each unit peaked. As illustrated in the histogram in Figure 5A, there appeared to be at least two peaks in the distribution. However, we could not rule out the 


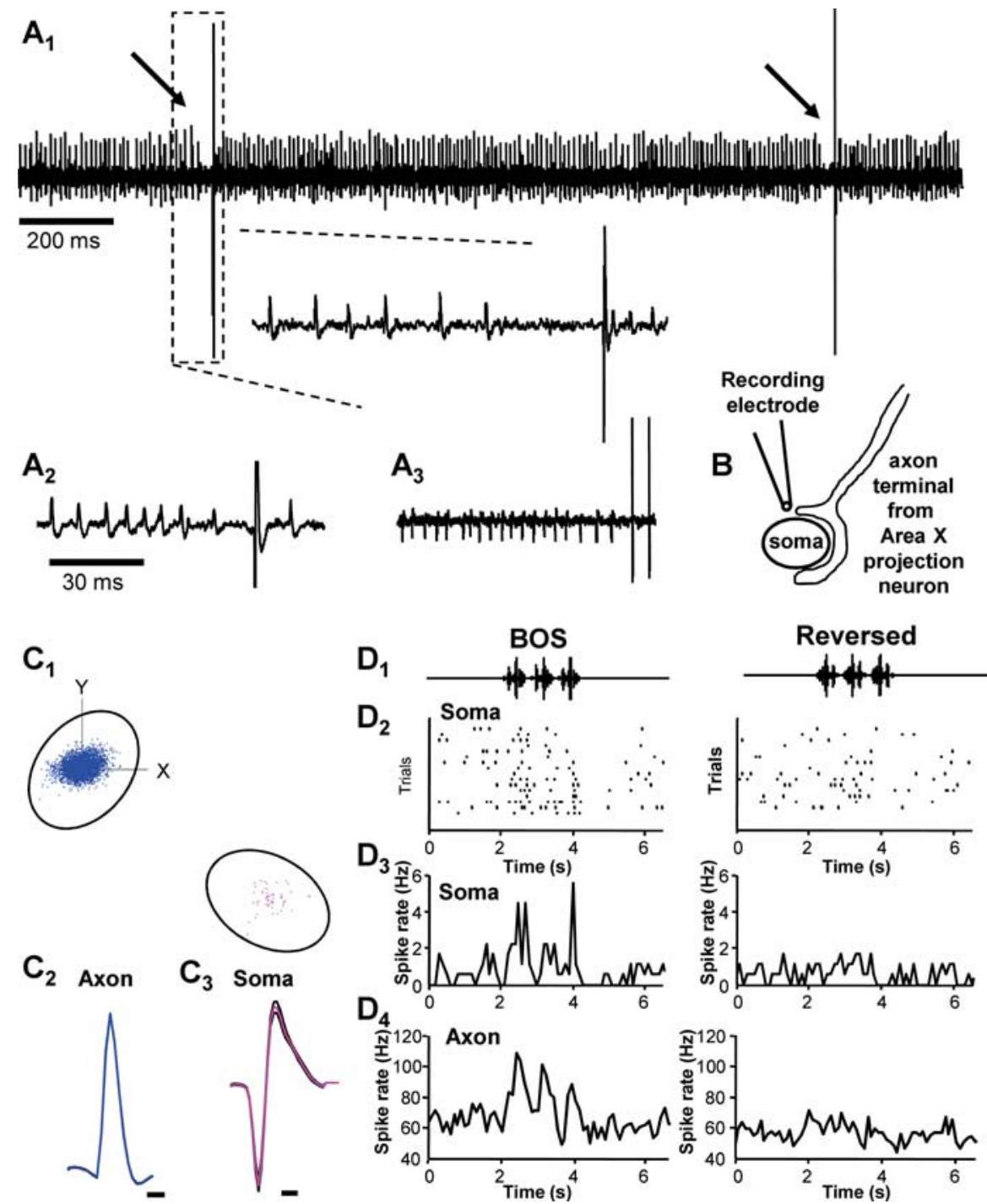

Figure 7. Paired terminal and soma recordings. $A_{1}-A_{3}$, Three examples of simultaneous recordings of HF and LF units in DLM. Two discriminable units with characteristic spike waveforms could be recorded simultaneously on single electrodes. $A_{1}$, A large soma spike occurring after a brief pause in HF terminal activity (small spikes) during a baseline period. $A_{2}, A_{3}$, Further illustration from different paired recordings of a characteristically small, monophasic $\mathrm{HF}$ terminal spike paired with large, biphasic soma spikes. The occasional change in terminal spike amplitude termporally close to somatic spikes is hypothesized to be attributable to changes in presynaptic firing rate about the soma spike (see Results). $\boldsymbol{B}$, Schematized representation of the hypothesized recording arrangement, with the electrode tip positioned very close to both the cell soma and the axon terminal. $\boldsymbol{C}_{1}-\boldsymbol{C}_{3}$, Principal component analysis of spike shape used for paired-recording spike sorting. $C_{1}$, An example of principal component clusters on spike shape parameters. Clusters were completely separate in all pairs recorded. $C_{2}$, The average waveform (with mean $\pm 1 S D$ in black) of the $\mathrm{HF}$ unit from the cluster shown above it. Calibration, $0.25 \mathrm{~ms}$. $C_{3}$, The average waveform of the LF unit in the cluster above it (with mean $\pm 1 S D$ in black). Calibration, $0.3 \mathrm{~ms}$. $\boldsymbol{D}_{1}-\boldsymbol{D}_{4}$, Auditory responses in a paired recording. $\boldsymbol{D}_{1}, 0$ scillograms of BOS and Rev stimuli indicating the time of playback per sweep. $\boldsymbol{D}_{2}$, Raster plot of LF unit within the pair shown in $\boldsymbol{C} . \boldsymbol{D}_{\mathbf{3}^{\prime}}$ Peristimulus time histogram summarizing the auditory response of the LF unit to BOS and Rev across trials. $D_{4}$, Peristimulus time histogram of the HF unit paired with the LF unit above summarizing its auditory responses across trials.

possibility that this range represents a continuum, and results of juxtacellular labeling did not reveal diverse morphological cell classes that could give rise to the variety of responses. Although not explicitly studied, long-latency auditory responses can be seen in other song-system nuclei (Doupe, 1997; Rauske et al., 2003); thus, long-latency responses in DLM may reflect upstream auditory processing.

Across the population of LF units, the RS to BOS was significantly greater than to $\operatorname{Rev}(p<0.01$, paired $t$ test). These RS measurements were next used to quantify the potential of a single cell to discriminate between BOS and Rev with a $d^{\prime}$ metric. $d^{\prime}$ values describe the degree of discriminability of neural response to two stimuli, in our case BOS and Rev. The cumulative distribution of $d^{\prime}$ values is plotted in Figure $5 B$ for all HF and LF neurons. LF neurons inhibited by song (Fig. $5 B$, filled circles) demonstrated less selectivity but also accounted for some negative $d^{\prime}$ values, indicating greater inhibition during forward BOS than Rev.

Rapid spike-rate decelerations occur in pallidal-like axons during song playback

Basal ganglia output is hypothesized to use pauses in HF spontaneous activity to relay information through the thalamus (Albin et al., 1989; DeLong, 1990). In apparent conflict with this model, the HF pallidallike terminals we recorded displayed dramatic increases in firing rate during song playback (Figs. $4 A, 6 A$ ).

To examine how the basal ganglia might signal through thalamus, we carefully analyzed the patterns of HF unit firing. We first considered the possibility that long ISIs persisted during song playback, despite an overall firing rate increase; such long ISIs could disinhibit thalamic targets. To examine this, we analyzed ISI distributions in the pallidal units during BOS playback and baseline periods (Fig. 6B). ISI distributions during BOS playback were consistently shifted toward shorter ISIs compared with the baseline period (Fig. $6 B)$. The right-hand tail of the ISI distributions, indicating the longest ISIs, did not differ between baseline and playback conditions, suggesting no systematic difference in the distribution of long ISIs between baseline periods and BOS playback (Fig. $6 B$, inset). To better characterize the right-hand tail of the ISI distributions, we next asked whether the longest ISIs per sweep occur more frequently during song playback than during baseline, although at a frequency not visible in an overall ISI probability function. We calculated the frequency (occurrences per second) of the longest ISIs during each sweep over baseline and playback epochs (Fig. 6C). The longest ISIs, defined as those that are longer than $95 \%$ of all ISIs (corresponding to a $26.4 \pm 1.0 \mathrm{~ms}$ ISI threshold), were not significantly more frequent during BOS than during baseline periods (Fig. $6 D)(p>0.5$, paired $t$ test). Furthermore, contrary to the prediction that the longest ISIs might occur during BOS playback, the longest ISIs during baseline periods (defined as those in the 95th percentile bracket) were, on average, $1.5 \pm 0.4 \mathrm{~ms}$ longer than those during BOS playback. These results indicate that pallidal inputs to DLM do not display obvious long "pauses" while sensory information is being relayed through the thalamus.

If pallidal disinhibition of thalamus is not evident in either the 
overall firing rate or the presence of long ISIs occurring during sensory stimuli, what could account for thalamic activity during epochs of increased inhibition? DLM neurons can spike after the rate of pallidal afferents drops in the absence of excitatory inputs in vitro (Person and Perkel, 2005). Thus, we next analyzed HF units for periods of rapid firing rate deceleration (see Materials and Methods). Briefly, we took the derivative of the instantaneous firing rate and drew a 5\% threshold cutoff (Fig. 6E). Next, we calculated the time of each downward threshold crossing, indicating firing rate deceleration, across trials, and summarized them for each cell (Fig. $6 F$ ). Across the population of HF units, there were consistently more rapid firing rate decelerations during playback than during baseline periods (Fig. $6 G)(p<0.0001$, paired $t$ test; $n=$ 37 ), raising the possibility that this feature in pallidal activity could be relayed through the thalamus.

In summary, we found that pallidallike terminals increase in firing rate during song playback and do not display long pauses during sensory stimuli. However, the firing rate of pallidal-like terminals is rapidly modulated during song playback, leading to epochs of rapid firing rate decelerations that occur most frequently during song playback.

\section{Simultaneous $\mathrm{HF}$ and LF recordings}

The specialized arrangement of presynaptic and postsynaptic neural elements in DLM raised the possibility that we might be able to record simultaneously from pairs of synaptically connected units on a single electrode (i.e., from both presynaptic and postsynaptic elements). Because we found that the HF units in DLM are axon terminals with distinct firing rates, waveforms, and auditory response profiles from LF neurons, we reasoned that we could classify pairs of recorded neurons into HF or LF categories unambiguously. In fact, such phenomenology has been reported and studied in the mammalian LGN, where presynaptic action potentials and/or subthreshold postsynaptic potentials can be detected extracellularly and are termed "prepotentials" or "slow potentials" (Hubel and Wiesel, 1961; Kaplan and Shapley, 1984; Sincich et al., 2007). In such experiments, pairs are discriminable only when the LF-unit signal-to-noise ratio is very high, typically over 15:1 (Sincich et al., 2007). To aid in this degree of isolation, we used high-impedance electrodes, requiring very close apposition of the recording electrode to the recorded neuron to detect any neural signal. In 11 cases, we recorded such pairs (Fig. 7A). These recordings were typified by a smaller-amplitude HF unit coupled with a large-amplitude LF unit (Fig. $7 A_{1}-A_{3}$ ). The dramatic difference in amplitudes between the HF and LF unit could result from the hypothesized recording configuration illustrated in Figure $7 B$, where the recording electrode was very close to the soma but could still detect axon terminal spiking. For all pairs, clusters of principal components from spike waveforms of the two units (calculated with Spike2 software) were completely separate, leading to unambig- uous assignment of spikes to either class (Fig. $7 C_{1}-C_{3}$ ). We note that the spike width of HF units in pairs was significantly longer than the spike width of singly isolated HF units $(1.23 \pm 0.12 \mathrm{vs}$ $0.41 \pm 0.02 \mathrm{~ms}$, respectively; $p<0.001$ ), suggesting that the HF units recorded in pairs may include a late component from the postsynaptic potential. We refer to this type of activity as axon terminal spiking to reflect this possibility.

We isolated single units from paired recordings off-line using spike-sorting software (see Materials and Methods) and analyzed them as singly isolated units described above. The HF units in pairs had ISI distributions that were typical of singly isolated HF units described above (Fig. $6 \mathrm{H}$ ). HF units in pairs had an average rate of $67 \pm 3.3 \mathrm{~Hz}$ (range, $57.25-86.5 \mathrm{~Hz}$ ), and LF units in pairs had an average rate of $2.28 \pm 0.63 \mathrm{~Hz}$. These ranges of firing rates fell squarely within the distributions of average spontaneous rates reported for singly isolated units (Fig. 2), supporting the conclusion that our off-line isolation included only the two paired HF and LF units.

Our paired pallido-thalamic recordings allowed us to investigate the feature of pallidal input that preceded postsynaptic thalamic spiking. We calculated a thalamic STA by cross-correlating the pallidal input with somatic output. For this analysis, axon terminal spikes occurring within $250 \mathrm{~ms}$ of each somatic spike were aligned with reference to each somatic spike. Analyses of this sort revealed a distinct gap in the raster plot of axon terminal spikes just before the soma spike in all pairs tested (Fig. 8A,B). The STA was asymmetric for all pairs, consistent with the idea that we were recording from both a presynaptic and postsynaptic element with a functional connection (Gerstein and Perkel, 

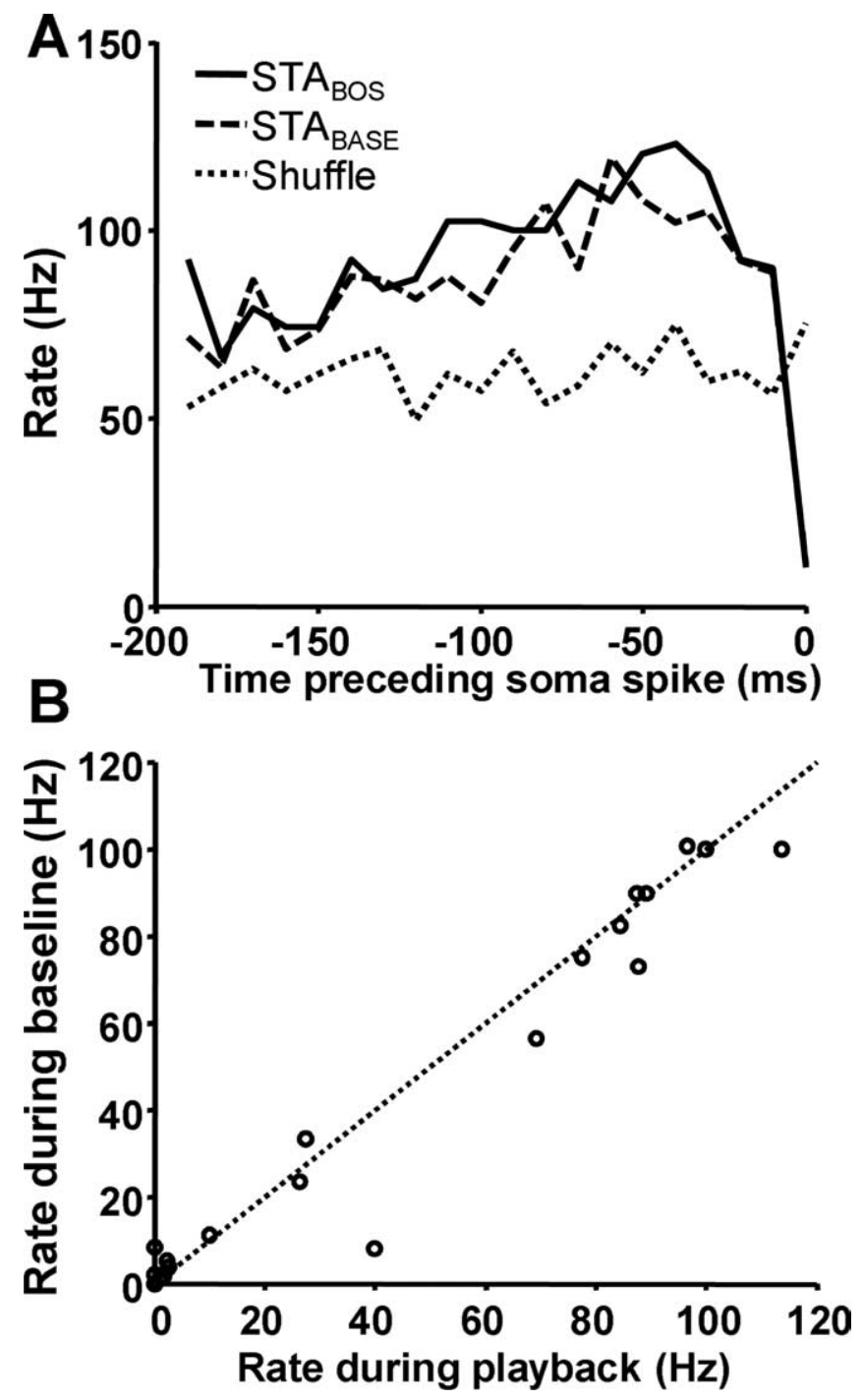

Figure 9. No difference in axon terminal spike patterns preceding soma spikes between $B O S$ playback or baseline periods. $\boldsymbol{A}$, Example STA illustrating no difference in pattern of activity preceding spikes that occur during song playback (solid lines) or during baseline periods (dashed lines). These features are not random, because shuffled spike times do not return the same feature (dotted line). $\boldsymbol{B}$, Rates of the STA $\mathrm{BASE}_{\text {and }}$ STA $\mathrm{A}_{\mathrm{BOS}}$ were compared at two time points ( $0-10$ and $11-20 \mathrm{~ms}$ before soma spike) for all pairs. No systematic difference between STA $_{\text {BASE }}$ and STA $_{\text {BOS }}$ was observed.

1969). We note the caveat that these data do not directly prove that the pairs we recorded were synaptically coupled.

In the previous section, we described ISI distributions of singly isolated HF units. In those units, we found no evidence of long ISIs occurring more frequently during song playback. However, the raster plots of axon terminal spikes aligned to somatic spikes always displayed a gap when axon terminal spikes were aligned to the occurrence of the somatic spike. To reconcile these two findings, we analyzed the duration of the gap in the STAs occurring before somatic spikes. Across pairs, this interval averaged $16.5 \pm$ $1.9 \mathrm{~ms}$ ( $n=11$ pairs; 3399 somatic spikes; gap before shuffled spikes was significantly shorter: $10.6 \pm 0.62 \mathrm{~ms} ; p<0.01$, paired $t$ test). This "gap" of $\sim 16 \mathrm{~ms}$ seen preceding LF spikes fell near the mean of ISIs, however, and thus did not reflect "long" ISIs. In fact, a pause of $\sim 16 \mathrm{~ms}$ corresponds to an instantaneous firing rate of $\sim 60 \mathrm{~Hz}$, near the mean of the baseline firing rates (Fig. $6 H$ ). In addition, as was seen in analyses of individually isolated
HF units, long pauses did not occur more frequently during song playback than baseline periods $(p>0.05)$.

We next analyzed a broader envelope of axon terminal spike rates preceding somatic spikes, rather than focusing only on the final pre-somatic spike interval. We summarized each STA with a histogram reflecting the average presynaptic firing rate before each soma spike and averaged them across cells (Fig. 8C). In most cases ( 8 of 11), the STAs revealed a distinct increase in average firing rate of the pallidal neuron $50 \mathrm{~ms}$ before the somatic spike to an average rate of $89.9 \pm 9.0 \mathrm{~Hz}$ (Fig. $8 \mathrm{D}$ ) [mean change from baseline rate, $22 \mathrm{~Hz}$ (range, $-11-92 \mathrm{~Hz}$ )]. This overall increase was followed by a rate decrease to $10.3 \pm 6.7 \mathrm{~Hz}$ during the $10 \mathrm{~ms}$ before the somatic spike (Fig. $8 D$ ). This change in rate corresponded to a deceleration of $79.6 \pm 10.1 \mathrm{~Hz}$. In addition, as in singly isolated HF neurons, rate decelerations in HF units recorded in pairs occurred significantly more frequently during song playback than during baseline periods $(p<0.005$, paired $t$ test). Therefore, together, these results suggest that pallidal neurons could signal through the thalamus by exhibiting decelerations in firing rate that in many cases immediately follow firing rate increases.

Our paired recordings allowed us to address the extent to which projections to DLM from sources other than Area X contribute to auditory responses. Specifically, we wondered whether other inputs might contribute to DLM firing during song. If so, we reasoned that the STA for spikes occurring during playback would be different from the STA for spikes occurring during baseline periods, reflecting an altered relationship between Area $\mathrm{X}$ and DLM. Thus, we directly compared the STA calculated from spikes that occurred during BOS $\left(\mathrm{STA}_{\mathrm{BOS}}\right)$ with the STA calculated from spikes that occurred during baseline periods (STABASE). We found no difference in the spike rate of the pallidal axon terminal before somatic spikes during BOS playback or baseline periods (Fig. 9A). We analyzed this across pairs by matching $\mathrm{STA}_{\mathrm{BOS}}$ with $\mathrm{STA}_{\mathrm{BASE}}$ at two time points before the somatic spike trigger. We chose times in the STA immediately before spiking, when the greatest degree of rate modulation occurred, specifically between 0 and $10 \mathrm{~ms}$ and 11 and $20 \mathrm{~ms}$ before the soma spike. Values of $\mathrm{STA}_{\mathrm{BOS}}$ and $\mathrm{STA}_{\mathrm{BASE}}$ at these time points were not significantly different (Fig. $9 B)(p>0.1$, paired $t$ test). Together, these data suggest that the specific changes in presynaptic firing patterns in Area X neurons projecting to DLM, as revealed in the STA, may be necessary for, and could cause, thalamic spiking.

\section{Discussion}

Our investigation of DLM unit activity in anesthetized zebra finches yielded a window into the process of basal ganglia-pallidal relay through the thalamus. As expected, we found that thalamic relay neurons respond selectively to playback of BOS, a property that has been studied extensively in the primary afferent and efferent nuclei of DLM (Doupe and Konishi, 1991; Doupe, 1997; Solis and Doupe, 1997; Rosen and Mooney, 2000). Unexpectedly, we also found a population of HF units in DLM that have robust, selective auditory responses. Pharmacological and anatomical evidence suggests that these units are giant GABAergic synaptic terminals or axons from Area X pallidal-like neurons terminating in DLM (Fig. 3). These units were excited by song, surging from $50 \mathrm{~Hz}$ up to instantaneous rates of $700 \mathrm{~Hz}$ (Fig. $6 \mathrm{~B}$ ). In a few cases, we successfully recorded from the presynaptic and postsynaptic elements simultaneously (Fig. 7). This direct observation of simultaneous pallidal activity and thalamic output allowed us to uncover a correlation between pallidal and thalamic activity. We found that rather than simple pauses, pallidal units 
tended to show more complex changes in firing rate before thalamic neuron spiking that include rapid decelerations (Figs. 6, 8 ). These data add clarity to models of pallido-thalamic relay to downstream pallial structures.

The AFP in oscine songbirds is required for song learning in juveniles and for song plasticity in adults (Bottjer et al., 1984; Sohrabji et al., 1990; Scharff and Nottebohm, 1991; Williams and Mehta, 1999; Brainard and Doupe, 2000a,b; Kao et al., 2005; Ölveczky et al., 2005). The output of the AFP can influence song structure in real time (Kao et al., 2005) and displays firing that is time locked to stereotyped features of song (Leonardo, 2004). Because the AFP includes a basal ganglia circuit, general mechanistic questions about how basal ganglia signals reach the pallium, a forebrain area of which mammalian cortex is a part, can be addressed, with the advantage that the neural circuitry under investigation is dedicated to a specific behavior.

The textbook model of basal ganglia relay through thalamus (Fig. $10 \mathrm{~A}$ ) was not supported in this study. Specifically, pallidal output neurons are hypothesized to signal by pausing during HF spontaneous activity (Hikosaka and Wurtz, 1983; Deniau and Chevalier, 1985). In contrast, we found that pallidal afferents to DLM markedly increase their firing rates in response to song playback. These neurons likely correspond to HF units recorded in Area X in previous studies (Doupe, 1997; Solis and Doupe, 1997, 1999; Margoliash, 1997; Hessler and Doupe, 1999), with RS and $d^{\prime}$ measurements comparable to those reported previously. Auditory responses among the different neuronal types within Area X have not been published, thus specific comparisons between our data and those reported elsewhere are premature. Nevertheless, because we recorded the pallidal axon terminals, we could be certain that these neurons represented the output of the basal ganglia to the thalamus. Increases in pallidal firing rate have been seen in the GPi of primates (Anderson and Horak, 1985; DeLong et al., 1985; Turner and Anderson, 1997), but this phenomenon has been hypothesized to provide lateral inhibition onto target thalamic neurons (Nambu et al., 2002). In the present study, paired recordings of pallidal axon terminals and thalamic neurons allowed us to observe pallidal signaling during target thalamic neuron activation. In these cases, we observed that the firing rate of the pallidal neuron increased whereas the thalamic neuron firing rate also increased. Because this projection is GABAergic and strongly inhibitory (Luo and Perkel, 1999a,b; Person and Perkel, 2005), this scheme was counterintuitive and does not support the hypothesis of lateral inhibition. Rather, the increases in firing rate seen in the pallidal neurons during song playback could enhance the effect of subsequent decreases in firing rate, which could in turn drive action potentials that propagate through the thalamus (Person and Perkel, 2005).

Our experiments provide evidence for thalamic relay of basal ganglia output that uses rapid modulation of firing rate to produce spike-rate decelerations that may be relayed through tha-
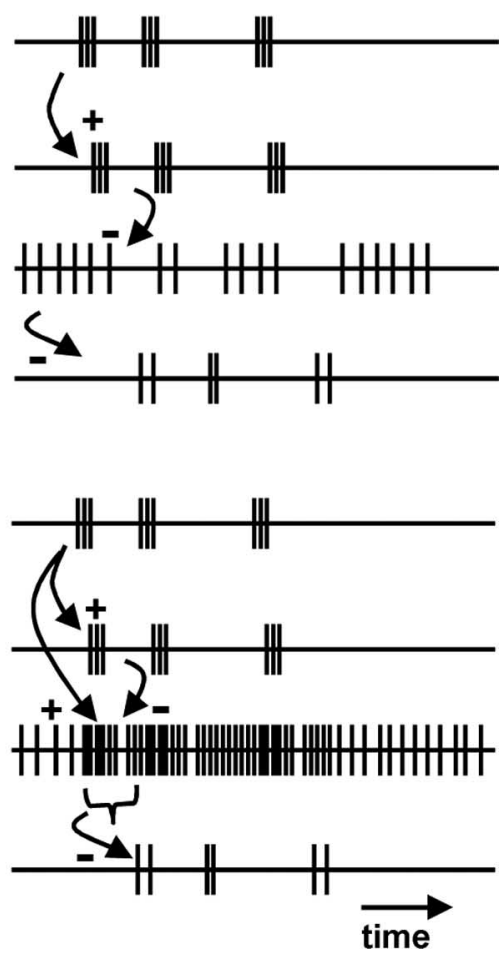

Figure 10. Working models of two modes of information propagation through a basal ganglia-thalamo-pallial loop. $\boldsymbol{A}$, The _X) may drive phasic increases in pallidal projection neuron (Area X_PN) firing rates. This pallidal neuron bursting could be further shaped by striatal-mediated inhibition (Area X_SN), creating a deceleration feature that may drive thalamic

lamic neurons (Fig. 10B) (Person and Perkel, 2005). Such patterns of activity could be created by bursting excitatory inputs [possibly originating in the telencephalic song nucleus HVC (Kozhevnikov and Fee, 2007)] driving bursting in pallidal-like output neurons in Area X (Farries et al., 2005) above their ongoing HF spontaneous activity. Spiny neurons activated by HVC inputs could further shape pallidal-like neuron busting by providing disynaptic inhibition onto pallidal projection neurons, attenuating the increased firing rates (Fig. $10 \mathrm{~B}$ ). Such a scheme could enhance a deceleration in spike rate. In fact, a rapid decrease in the occurrence of presynaptic spikes before postsynaptic spikes was strikingly reminiscent of the feature in IPSP trains found to drive rebound firing in vitro (Person and Perkel, 2005) and is consistent with such a mechanism driving spiking in vivo. The pallidal-like output neurons in Area X are likely the AF neurons recorded in vitro that have been found to receive direct excitatory inputs, as have spiny neurons (Farries and Perkel, 2002; Farries et al., 2005).

Hypotheses regarding the mechanism of basal ganglia signal relay through thalamus have implied that disinhibition of thalamus gates excitatory inputs from the cortex, which drive thalamic neurons to spike (Deniau and Chevalier, 1985). In contrast, previous experiments in a slice preparation of DLM have shown that GABAergic synaptic transmission alone can drive activity by eliciting reliable postinhibitory rebound spikes (Luo and Perkel, 1999b; Person and Perkel, 2005). Furthermore, DLM neurons in vitro respond to a precise statistical feature in HF GABAergic trains of IPSPs. The STA describing axonal input to thalamic neurons calculated in vivo closely resembles the IPSP patterns 
found to drive postinhibitory rebound in vitro (Person and Perkel, 2005). Thus, data presented here are consistent with postinhibitory rebound-mediated drive, which could serve as the mechanism for basal ganglia relay through thalamus in the songbird AFP. However, it is important to recall that AMPA receptormediated EPSPs have been observed in DLM neurons (Luo and Perkel, 1999b), suggesting that more complex circuitry may influence DLM relay under certain conditions. In addition, neuromodulators acting on the thalamus may influence resting membrane potential to alter the mode of basal ganglia relay (McCormick and Pape, 1990).

Lesions of Area X in juvenile songbirds lead to an increase in overall variability of song output (Scharff and Nottebohm, 1991) in line with destabilization effects seen with substantia nigra pars reticulata inactivation and GPi inactivation in mammals (Hikosaka and Wurtz, 1985; Inase et al., 1996). Lesions of the AFP output nucleus LMAN do the opposite; song variability is nearly abolished. Both manipulations prohibit normal song copying (Bottjer et al., 1984; Scharff and Nottebohm, 1991). Little is known about the effect of Area X inactivation on DLM activity. If Area X can drive activity precisely under certain conditions and simply gate activity under other conditions, DLM lesions may point to a diverse role for the thalamus in processing signals from the telencephalon. Future experiments should explore more directly the role of specific neurotransmitters and modulators involved with controlling DLM unit activity in vivo.

Do our data shed any light on general mechanisms of basal ganglia relay through diencephalic targets? Patterns of GPi activity in patients with Parkinson's disease show heightened levels of bursts and pauses, similar in structure to the pallidal feature observed to precede thalamic spikes reported here (Hashimoto et al., 2003). An effective therapeutic strategy is HF stimulation of the GPi or the STN, which, contrary to initial explanations, is now thought to increase pallidal spike rates and abolish pathological bursting activity in the GPi, establishing a more regular firing rate (Hashimoto et al., 2003; Bar-Gad et al., 2004). If healthy GPi uses a deceleration-based pattern of activity to relay sensorimotor information through thalamus, it is possible that the increased rate of bursting seen in the GPi of Parkinson's disease patients obscures the similarly structured movement-related signaling. Future models of basal ganglia function may better model pathologies by incorporating new information about these complexities of pallidothalamic interactions.

\section{References}

Albin RL, Young AB, Penney JB (1989) The functional anatomy of basal ganglia disorders. Trends Neurosci 12:366 -375 .

Anderson ME, Horak FB (1985) Influence of the globus pallidus on arm movements in monkeys. III. Timing of movement-related information. J Neurophysiol 54:433-448.

Anderson ME, Turner RS (1991) Activity of neurons in cerebellar-receiving and pallidal receiving areas of the thalamus of the behaving monkey. J Neurophysiol 66:879-893.

Bar-Gad I, Elias S, Vaadia E, Bergman H (2004) Complex locking rather than complete cessation of neuronal activity in the globus pallidus of a 1-methyl-4-phenyl-1,2,3,6-tetrahydropyridine-treated primate in response to pallidal microstimulation. J Neurosci 24:7410-7419.

Bottjer SW, Miesner EA, Arnold AP (1984) Forebrain lesions disrupt development but not maintenance of song in passerine birds. Science 224:901-903.

Brainard MS, Doupe AJ (2000a) Interruption of a basal ganglia-forebrain circuit prevents plasticity of learned vocalizations. Nature 404:762-766.

Brainard MS, Doupe AJ (2000b) Auditory feedback in learning and maintenance of vocal behaviour. Nat Rev Neurosci 1:31-40.

DeLong MR (1990) Primate models of movement disorders of basal ganglia origin. Trends Neurosci 13:281-285.
DeLong MR, Crutcher MD, Georgopoulos AP (1985) Primate globus pallidus and subthalamic nucleus: functional organization. J Neurophysiol 53:530-543.

Deniau JM, Chevalier G (1985) Disinhibition as a basic process in the expression of striatal functions. II. The striato-nigral influence on thalamocortical cells of the ventromedial thalamic nucleus. Brain Res 334:227-233.

Doupe AJ (1997) Song- and order-selective neurons in the songbird anterior forebrain and their emergence during vocal development. J Neurosci 17:1147-1167.

Doupe AJ, Konishi M (1991) Song-selective auditory circuits in the vocal control system of the zebra finch. Proc Natl Acad Sci USA 88:11339-11343.

Doupe AJ, Perkel DJ, Reiner A, Stern EA (2005) Birdbrains could teach basal ganglia research a new song. Trends Neurosci 28:353-363.

Farries MA, Perkel DJ (2002) A telencephalic nucleus essential for song learning contains neurons with physiological characteristics of both striatum and globus pallidus. J Neurosci 22:3776-3787.

Farries MA, Ding L, Perkel DJ (2005) Evidence for "direct" and "indirect" pathways through the song system basal ganglia. J Comp Neurol 484:93-104.

Gerstein GL, Perkel DH (1969) Simultaneously recorded trains of action potentials: analysis and functional interpretations. Science 164:828 -830.

Green DM, Swets JA (1966) Signal detection theory and psychophysics. New York: Wiley.

Hashimoto T, Elder CM, Okun MS, Patrick SK, Vitek JL (2003) Stimulation of the subthalamic nucleus changes the firing pattern of pallidal neurons. J Neurosci 23:1916-1923.

Havey DC, Caspary DM (1980) A simple technique for constructing "piggyback" multibarrel microelectrodes. Electroencephalogr Clin Neurophysiol 48:249-251.

Hessler NA, Doupe AJ (1999) Singing-related neural activity in a dorsal forebrain-basal ganglia circuit of adult zebra finches. J Neurosci 23:10461-10481.

Hikosaka O, Wurtz RH (1983) Visual and oculomotor functions of monkey substantia nigra pars reticulata. IV. Relation of substantia nigra to superior colliculus. J Neurophysiol 49:1285-1301.

Hikosaka O, Wurtz RH (1985) Modification of saccadic eye movements by GABA-related substances. II. Effects of muscimol in monkey substantia nigra pars reticulata. J Neurophysiol 53:292-308.

Hubel DH, Wiesel TN (1961) Integrative action in the cat's lateral geniculate body. J Physiol (Lond) 155:385-398.

Inase M, Buford JA, Anderson ME (1996) Changes in control of arm position, movement, and thalamic discharge during local inactivation in the globus pallidus of the monkey. J Neurophysiol 75:1087-1104.

Janata P, Margoliash D (1999) Gradual emergence of song selectivity in sensorimotor structures of the male zebra finch song system. J Neurosci 19:5108-5118.

Kao MH, Doupe AJ, Brainard MS (2005) Contributions of an avian basal ganglia-forebrain circuit to real-time modulation of song. Nature 433:638-643.

Kaplan E, Shapley R (1984) The origin of the S (slow) potential in the mammalian lateral geniculate nucleus. Exp Brain Res 55:111-116.

Kopp-Scheinpflug C, Dehmel S, Dorrscheidt GJ, Rubsamen R (2002) Interaction of excitation and inhibition in anteroventral cochlear nucleus neurons that receive large endbulb synaptic endings. J Neurosci 22:11004-11018.

Kozhevnikov AA, Fee MS (2007) Singing-related activity of identified HVC neurons in the zebra finch. J Neurophysiol 97:4271-4283.

Leonardo A (2004) Experimental test of the birdsong error-correction model. Proc Natl Acad Sci USA 101:16935-16940.

Luo M, Perkel DJ (1999a) Long-range GABAergic projection in a circuit essential for vocal learning. J Comp Neurol 403:68-84.

Luo M, Perkel DJ (1999b) A GABAergic, strongly inhibitory projection to a thalamic nucleus in the zebra finch song system. J Neurosci 19:6700-6711.

Luo M, Perkel DJ (2002) Intrinsic and synaptic properties of neurons in an avian thalamic nucleus during song learning. J Neurophysiol 88:1903-1914.

Luo M, Ding L, Perkel DJ (2001) An avian basal ganglia pathway essential for vocal learning forms a closed topographic loop. J Neurosci 21:6836-6845. 
Margoliash D (1997) Functional organization of forebrain pathways for song production and perception. J Neurobiol 33:495-500.

McCormick DA, Pape HC (1990) Noradrenergic and serotonergic modulation of a hyperpolarization-activated cation current in thalamic relay neurones. J Physiol (Lond) 431:319-342.

Nambu A, Tokuno H, Takada M (2002) Functional significance of the cortico-subthalamo-pallidal "hyperdirect" pathway. Neurosci Res 43:111-117.

Okuhata S, Saito N (1987) Synaptic connections of thalamo-cerebral vocal nuclei of the canary. Brain Res Bull 18:35-44.

Ölveczky BP, Andalman AS, Fee MS (2005) Vocal experimentation in the juvenile songbird requires a basal ganglia circuit. PLoS Biol 3:e153.

Person AL, Perkel DJ (2005) Unitary IPSPs drive precise thalamic spiking in a circuit required for learning. Neuron 46:129-140.

Pinault D (1996) A novel single-cell staining procedure performed in vivo under electrophysiological control: morpho-functional features of juxtacellularly labeled thalamic cells and other central neurons with biocytin or Neurobiotin. J Neurosci Methods 65:113-136.

Rauske PL, Shea SD, Margoliash D (2003) State and neuronal classdependent reconfiguration in the avian song system. J Neurophysiol 89:1688-1701.

Rosen MJ, Mooney R (2000) Intrinsic and extrinsic contributions to auditory selectivity in a nucleus critical for vocal plasticity. J Neurosci 20:5437-5448.

Scharff C, Nottebohm F (1991) A comparative study of the behavioral def- icits following lesions of various parts of the zebra finch song system: implications for vocal learning. J Neurosci 11:2896-2913.

Sincich LC, Adams DL, Economides JR, Horton JC (2007) Transmission of spike trains at the retinogeniculate synapse. J Neurosci 27:2683-2692.

Sohrabji F, Nordeen EJ, Nordeen KW (1990) Selective impairment of song learning following lesions of a forebrain nucleus in the juvenile zebra finch. Behav Neural Biol 53:51-63.

Solis MM, Doupe AJ (1997) Anterior forebrain neurons develop selectivity by an intermediate stage of birdsong learning. J Neurosci 17:6447-6462.

Solis MM, Doupe AJ (1999) Contributions of tutor and bird's own song experience to neural selectivity in the songbird anterior forebrain. J Neurosci 19:4559-4584.

Steriade M, Contreras D (1995) Relations between cortical and thalamic cellular events during transition from sleep patterns to paroxysmal activity. J Neurosci 15:623-642.

Turner RS, Anderson ME (1997) Pallidal discharge related to the kinematics of reaching movement in two dimensions. J Neurophysiol 77:1051-1074.

von Krosigk M, Bal T, McCormick DA (1993) Cellular mechanisms of a synchronized oscillation in the thalamus. Science 261:361-364.

Wang C, Cleland BG, Burke W (1985) Synaptic delay in the lateral geniculate nucleus of the cat. Brain Res 343:236-245.

Williams H, Mehta N (1999) Changes in adult zebra finch song require a forebrain nucleus that is not necessary for song production. J Neurobiol 39:14-28. 